\title{
Efeitos de programas de reabilitação multidisciplinar no tratamento de pacientes com doença de Alzheimer: uma revisão sistemática
}

\author{
Effects of multidisciplinary rehabilitation programs on \\ treatment of patients with Alzheimer's disease: a systematic review
}

\author{
Bruna Guimarães Madureira ${ }^{1}$, Maria Geralda Pereira², Patrick Roberto Avelino², \\ Henrique Silveira Costa², Kênia Kiefer Parreiras de Menezes²
}

\begin{abstract}
Resumo
Introdução: A Doença de Alzheimer (DA) é caracterizada por perda das funções cognitivas de forma progressiva, como falhas na memória, aprendizagem e linguagem, que tendem a se agravar com o avanço da doença. As ações multidisciplinares no paciente com DA têm como objetivo interferir positivamente no processo saúde-doença, por meio de uma abordagem integral aos indivíduos e familiares, intervindo com ações voltadas à realidade na qual estão inseridos. Objetivo: Realizar uma revisão sistemática sobre os efeitos da reabilitação multidisciplinar em pacientes com Doença de Alzheimer (DA). Método: Foram realizadas buscas nas bases MEDLINE, LILACS, PEDro, CINAHL e Web of Science, sem restrição de data ou de idioma de publicação. Os artigos foram avaliados pelo título, pelo resumo e, posteriormente, pelo texto completo, por dois autores independentes. A qualidade metodológica dos estudos experimentais selecionados foi avaliada de acordo com a escala PEDro. Resultados: Foram incluídos cinco estudos, de qualidade metodológica moderada (5,4 na escala PEDro), que evidenciaram que um programa multidisciplinar pode ser eficaz no tratamento de pacientes com DA, com melhoras significativas, principalmente, em sintomas neuropsiquiátricos, depressão e qualidade de vida. Para as demais medidas de desfecho investigadas, devido à presença de poucos estudos que encontraram efeitos positivos (nível de estresse, ansiedade, independência, atividades) ou de resultados conflitantes (cognição), não é possível determinar a eficácia dessa intervenção. Conclusão: Esta revisão sistemática evidenciou que um programa multidisciplinar pode ser eficaz no tratamento de pacientes com DA, com melhoras significativas, principalmente, em sintomas neuropsiquiátricos, depressão e qualidade de vida. No entanto, os resultados para cognição, nível de ansiedade, estresse, independência e realização de atividades não foram significativos ou foram conflitantes.
\end{abstract}

Palavras-chave: Doença de Alzheimer; terapêutica; equipe de assistência ao paciente.

\footnotetext{
Abstract

Background: Alzheimer's Disease (AD) is characterized by a progressive loss of cognitive functions, such as memory, learning and language deficits, which tend to be aggravated by the progression of the disease. Multidisciplinary actions in the patient with $A D$ aim to positively interfere in the health-disease process, through an integral approach to individuals and families, intervening with actions focused on the reality in which they are inserted. Objective: Conduct a systematic review of the literature investigating the effects of multidisciplinary rehabilitation programs on patients with Alzheimer's disease (AD). Method: A searches was conducted

${ }^{1}$ Fundação Comunitária de Ensino Superior de Itabira - Itabira (MG), Brasil.

${ }^{2}$ Universidade Federal de Minas Gerais (UFMG) - Belo Horizonte (MG), Brasil.

Trabalho realizado na Fundação Comunitária de Ensino Superior de Itabira - Itabira (MG), Brasil.

Endereço para correspondência: Kênia Kiefer Parreiras de Menezes - Av. Pres. Antônio Carlos, 6627 - Pampulha - CEP: 31270-901 - Belo Horizonte (MG), Brasil

- Email: keniakiefer@yahoo.com.br

Fonte de financiamento: nenhuma.

Conflito de interesses: nada a declarar.
}

Este é um artigo publicado em acesso aberto (Open Access) sob a licença Creative Commons Attribution, que permite uso, distribuição e reprodução em qualquer meio, sem restrições desde que o trabalho original seja corretamente citado. 
in the Medline, LILACS, Scielo, PEDro, and CINAHL databases with no restriction regarding language and year of publication. The articles were evaluated by title, abstract, and full text and, finally selected according to eligibility by two independent authors. The methodological quality of the experimental studies was evaluated using the PEDro scale. Results: Five studies of moderate methodological quality were included in the present systematic review. Results showed that multidisciplinary programs may be effective in the treatment of AD patients, with significant improvements mainly in neuropsychiatric symptoms, depression, and quality of life. For the other outcome measures investigated, because few studies with positive effects (stress level, anxiety, autonomy, and activity) or conflicting results (cognition) were found, it was not possible to determine the effectiveness of this intervention. Conclusion: This systematic review has shown that multidisciplinary programs can be effective in the treatment of patients with $A D$, with significant improvements mainly in neuropsychiatric symptoms, depression, and quality of life; however, results regarding cognition, levels of anxiety and stress, autonomy, and performance of activities were neither significant nor conflicting.

Keywords: Alzheimer's disease; treatment; multidisciplinary.

\section{INTRODUÇÃO}

O aumento da população idosa é um evento mundial ${ }^{1}$. Na América Latina, entre 1980 e 2025, deverá ocorrer um aumento de $271 \%$ da população total, enquanto o crescimento da população acima de 60 anos será de $412 \%{ }^{1}$. Esse fenômeno, por sua vez, leva a mudanças nos padrões de morbimortalidade, com o crescimento de doenças neurodegenerativas ou demências ${ }^{2}$. A demência, uma das principais doenças não transmissíveis da população idosa, tem apresentado um crescimento na sua prevalência nacional e mundial ${ }^{3}$. Em 2010, o número total de pessoas com demência no mundo foi estimado em 35,6 milhões e está projetado para quase dobrar a cada 20 anos, chegando a aproximadamente 115,4 milhões em $2050^{3}$.

Dos vários tipos de demência apresentados pela população idosa, a Doença de Alzheimer (DA) é a principal deles". A DA é caracterizada por perda das funções cognitivas de forma progressiva, como falhas na memória, aprendizagem e linguagem, que tendem a se agravar com o avanço da doença ${ }^{4}$. Assim, em sua fase leve, há uma diminuição no desempenho das tarefas de vida diária, mas o indivíduo ainda consegue realizar, de forma independente, as atividades básicas do dia a dia ${ }^{5}$. Já na fase moderada, ocorre um maior comprometimento intelectual, fazendo com que o paciente dependa mais de assistência para realizar atividades instrumentais e atividades básicas diárias. Por fim, na fase grave, o paciente, geralmente, fica acamado e pode apresentar dificuldades de deglutição, sinais neurológicos, incontinência urinária e fecal, sendo necessária uma assistência integral ${ }^{5}$.

Embora ainda não exista tratamento estabelecido que possa curar ou reverter a degeneração causada pela DA, algumas modalidades têm demonstrado capacidade de retardar e/ou até mesmo de estagnar, temporariamente, o avanço dos sintomas ${ }^{6-8}$. As intervenções não farmacológicas, por exemplo, representam um importante complemento ao tratamento, podendo reduzir ainda mais as despesas com a doença ${ }^{9}$. Diferentes abordagens têm sido propostas, tais como reabilitação cognitiva, terapia ocupacional, atividade física, musicoterapia, terapia artística, entre outras intervenções ${ }^{10,11}$. Todas essas opções visam aliviar os déficits cognitivos e as alterações de comportamento e melhorar a qualidade de vida do paciente e de sua família ${ }^{12}$. No entanto, por se tratar de uma doença que incide em diversas dimensões da vida do idoso/da família, essas intervenções devem ser trabalhadas de forma integrada, com a participação de diferentes profissionais da saúde, por meio de equipes multidisciplinares ${ }^{13}$.

As ações multidisciplinares no paciente com DA têm como objetivo interferir positivamente no processo saúde-doença, por meio de uma abordagem integral aos indivíduos e familiares, intervindo com ações voltadas à realidade na qual estão inseridos ${ }^{14}$. Nessa linha, apenas uma recente revisão integrativa da literatura avaliou as evidências disponíveis sobre o efeito das ações multidisciplinares na saúde ou no tratamento do idoso com DA, reportando resultados positivos ${ }^{4}$. No entanto, embora tais resultados sejam encorajadores, para que um tratamento possa ser indicado para sua aplicação na prática clínica, estes devem ser investigados por meio de revisões sistemáticas. As revisões sistemáticas são consideradas a melhor forma de sintetizar a informação existente sobre determinado tópico, pois são realizadas de acordo com um método de características sistemáticas e explícitas ${ }^{15,16}$. Esse método evita conclusões errôneas que podem ser tiradas com base apenas em resultados de um ensaio clínico ou em revisões não sistemáticas. Assim, ainda é necessária a realização de uma revisão sistemática da literatura que avalie os efeitos de um programa multidisciplinar no tratamento de pacientes com DA.

Dessa forma, o objetivo do presente estudo foi realizar uma revisão sistemática da literatura, investigando os efeitos de programas de reabilitação multidisciplinares em pacientes com DA, quando comparados a nenhum tratamento ou a outros tipos de intervenção.

\section{MÉTODO}

Este estudo é uma revisão sistemática da literatura, com o objetivo de avaliar os efeitos de programas de intervenção multidisciplinar no tratamento de pacientes com DA.

\section{Identificação dos estudos}

Para tal, foram realizadas buscas computadorizadas nas bases de dados MEDLINE, LILACS, PEDro, CINAHL e Web of Science (Apêndice A), sem restrição em relação ao idioma e ao ano de publicação do estudo. Os termos de busca utilizados, com estratégias específicas para cada base, foram: dementia, dement, Alzheimer, lewy body, rehabilitation, social environment, patient 
care management, combined, modalities, therapies, combined modality therapy, multimodal, multiprofissional, interdisciplinar, multidisciplinar and multidisciplinary care team. Os artigos foram buscados, avaliados pelo título, pelo resumo e, posteriormente, pelo texto completo e, enfim, selecionados em relação à elegibilidade, por dois autores independentes (BGM e MGP). Em caso de discordâncias, um terceiro avaliador (KKPM) foi o responsável pela decisão final. Também foi realizada uma busca manual em todas as listas de referências dos artigos incluídos, a fim de encontrar estudos que não tivessem retornado nas buscas nas bases de dados. Quando o artigo não estava disponível on-line gratuitamente, foi solicitada por e-mail uma cópia do texto completo ao autor.

\section{Critérios de inclusão}

Em relação aos participantes, foram incluídos estudos com indivíduos adultos, portadores da DA, sem restrição de idade, sexo ou tempo de diagnóstico. Em relação à intervenção, foram incluídos estudos que tenham realizado um programa de tratamento, incluindo duas ou mais áreas profissionais (fisioterapia, psicologia, medicina, terapia ocupacional, assistência social, enfermagem, nutrição etc.). Não houve critérios em relação às medidas de desfecho, sendo incluídos todos os estudos que tenham avaliado os efeitos em qualquer domínio de funcionalidade do indivíduo. Por fim, em relação ao design dos estudos, foram incluídos ensaios clínicos aleatorizados, quase aleatorizados ou controlados.

\section{Extração dos dados}

Todos os dados foram extraídos e dispostos em um formulário padrão, adaptado da Colaboração Cochrane. As informações extraídas dos estudos foram: características da amostra (idade, sexo, tamanho da amostra e tempo de diagnóstico), objetivos do estudo, protocolo de intervenção (áreas profissionais incluídas no programa de tratamento, tempo, duração e frequência da intervenção), instrumentos utilizados na avaliação (medidas de desfecho investigadas) e resultados encontrados. Quando informações necessárias não foram encontradas na versão publicada dos estudos, detalhes adicionais foram solicitados ao autor de correspondência por e-mail.

\section{Avaliação da qualidade metodológica dos estudos}

A qualidade metodológica dos estudos experimentais incluídos foi avaliada de acordo com a escala PEDro, descrita na base de dados Physiotherapy Evidence Database ${ }^{17}$. A escala, composta de 11 itens, foi desenvolvida para classificar a qualidade metodológica (validade interna e informações estatísticas) de ensaios clínicos aleatorizados. Cada item, exceto o $1^{\circ}$, contribui com um ponto para a pontuação total da escala, que varia de 0 a 10 pontos. Foi utilizada a pontuação dos estudos descrita no endereço eletrônico da base de dados. A pontuação dos estudos não incluídos na base de dados PEDro não pontuados foi realizada pelos autores deste estudo.

\section{RESULTADOS}

A busca eletrônica resultou em 2.364 artigos, dos quais 2.291 artigos foram excluídos após a leitura dos títulos, e 47, após a leitura dos resumos. Portanto, restaram 26 artigos selecionados para a leitura completa do texto. Após a leitura, cinco artigos foram incluídos nesta revisão, segundo os critérios de inclusão estabelecidos. A busca manual não retornou nenhum artigo. Assim, um total de cinco artigos foram incluídos e descritos no presente estudo ${ }^{18-22}$. As razões para exclusão dos demais estudos foram: não eram experimentais, não realizaram uma intervenção multidisciplinar ou não eram pacientes com

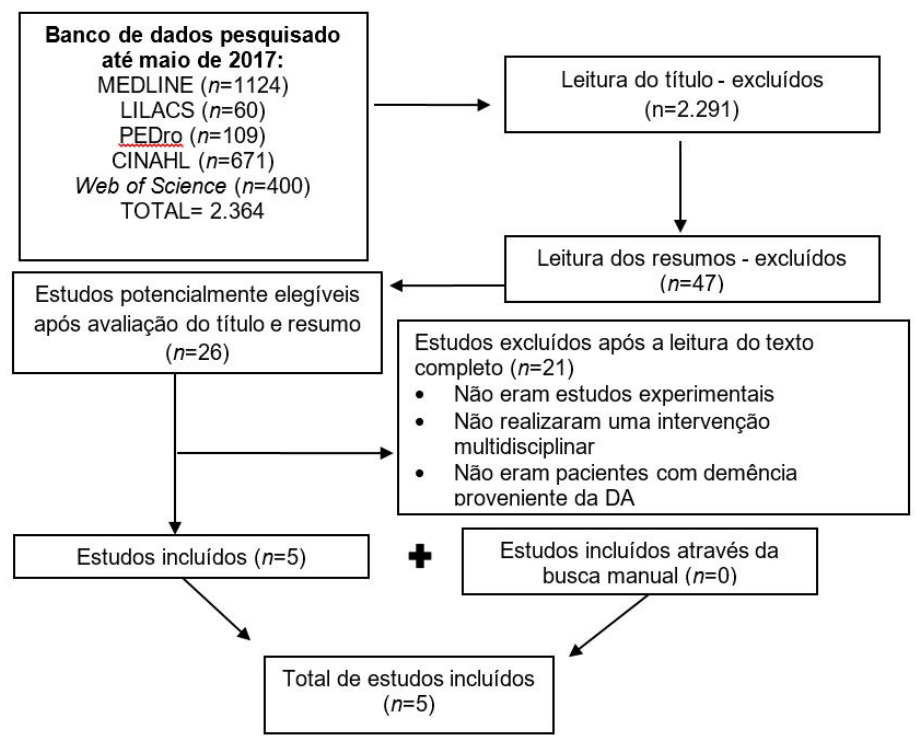

Figura 1. Fluxograma de inclusão e exclusão dos estudos 
demência proveniente da DA. A Figura 1 apresenta o fluxograma de seleção dos estudos, com cada etapa realizada.

Os estudos foram sumarizados na Tabela 1, sendo três ensaios controlados ${ }^{18,19,22}$, um ensaio clínico aleatorizado ${ }^{20} \mathrm{e}$ um quase aleatorizado ${ }^{21}$, com qualidade metodológica média de 5,4 pontos na escala PEDro (variação de 4 a 7) (Tabela 2). Todos os estudos foram realizados com amostra de conveniência e incluíram 576 participantes, com idade média de 77,7 anos, dos quais 236 eram homens. Dois estudos incluíram pacientes com DA leve ${ }^{21,22}$, um estudo incluiu pacientes com DA avançada ${ }^{20}$ e dois deles não especificaram a fase da doença em que os participantes se encontravam ${ }^{18,19}$. Apesar de os tratamentos terem como foco principal a multidisciplinaridade, três estudos não citaram os profissionais envolvidos, embora relatassem a aplicação de um protocolo multidisciplinar ${ }^{18,21,22}$. Dos demais estudos, as modalidades profissionais incluídas nas equipes multiprofissionais foram: enfermeiro, médico (neurologista), psicólogo e fisioterapeuta. Os protocolos de tratamento variaram de acompanhamentos semanais a anuais. Um estudo apresentou características diferentes dos demais, pois avaliou a eficácia, para os pacientes, da implementação "passo a passo" de um programa de intervenção multidisciplinar, comparado à aplicação de um programa também multidisciplinar, porém sem a implementação no método "passo a passo"20. As medidas de desfecho dos estudos incluíram sintomas neuropsiquiátricos (paciente e cuidador), depressão (paciente e cuidador), cognição, atividades da vida diária, autonomia, ansiedade (paciente e cuidador), estresse (paciente e cuidador), carga de cuidados (cuidador), agitação e qualidade de vida (paciente e cuidador).

\section{DISCUSSÃO}

Este trabalho teve como objetivo investigar os efeitos do tratamento multidisciplinar em paciente com DA. De forma geral, a abordagem multidisciplinar foi eficaz no tratamento de pacientes com DA. Os principais efeitos reportados pelos estudos foram melhora nos sintomas neuropsiquiátricos, na depressão, no nível de estresse, na agitação, na cognição, na autonomia, na carga do cuidador, na atividade de vida diária e na qualidade de vida. Além disso, dois estudos também avaliaram os sintomas nos cuidadores, reportando melhora dos níveis de depressão e de ansiedade.

Os sintomas neuropsiquiátricos, que são uma ampla gama de comportamentos angustiantes e de reações psicológicas, foram avaliados em quatro artigos, dos quais em três foi obtida melhora significativa do grupo experimental comparado ao grupo controle. É importante ressaltar que essas melhorias foram alcançadas sem aumentar significativamente o uso de antipsicóticos ou sedativo-hipnóticos ${ }^{18}$. Deixar os sintomas comportamentais e psicológicos dos pacientes de demência não tratados está associado ao esgotamento do cuidador, ao pior controle das comorbidades e ao excesso de custos com os cuidados de saúde ${ }^{23}$. Além disso, o tratamento farmacológico atual de sintomas comportamentais, como agressão ou psicose, é limitado pela eficácia modesta e pelos efeitos adversos importantes desses medicamentos ${ }^{18}$. Assim, ressalta-se a importância dos presentes achados deste estudo para a população com DA e seus cuidadores, evidenciando a importância e a melhora significativa de uma equipe variada e especializada no tratamento dessa condição.

A depressão também foi avaliada em todos os cinco artigos, obtendo melhora em três deles no grupo experimental para os pacientes ${ }^{18,20,21}$, enquanto um encontrou melhora dos sintomas depressivos nos cuidadores, quando comparados ao grupo controle ${ }^{22}$. Os resultados apontam que as consultas multidisciplinares e a reunião conjunta foram eficazes na redução dos sintomas depressivos ${ }^{18,20-22}$. Esses resultados são importantes, uma vez que a depressão é um dos sintomas mais frequentes da DA, podendo ser um dos primeiros sintomas da doença, antes mesmo da perda de memória ${ }^{24}$. Estudos anteriores apontam que mais de $90 \%$ das pessoas com Alzheimer tiveram alguma alteração desse tipo antes de demonstrarem os primeiros sinais de perda de memória e que indivíduos com DA tiveram duas vezes mais probabilidade de desenvolver depressão mais cedo do que aqueles sem demência ${ }^{24}$. Assim, encontrar soluções terapêuticas que produzam efeito significativo na redução desse sintoma nessa população, como o acompanhamento de uma equipe multidisciplinar, é fundamental para esses pacientes. Embora dois estudos não tenham encontrado diferenças significativas na redução da depressão após a intervenção, pode-se atribuir essa ausência de achados aos protocolos aplicados, uma vez que ambos foram os que apresentaram acompanhamento com menor intensidade e frequência com os pacientes ${ }^{18,19}$.

A cognição foi avaliada por quatro artigos ${ }^{18,19,21,22}$, dos quais somente um estudo ${ }^{21}$ encontrou melhora significativa no grupo experimental, quando comparado ao grupo controle para esse desfecho. No entanto, é importante enfatizar que o indivíduo com DA passa por um processo patológico de envelhecimento, definido como envelhecimento secundário, no qual ocorre degeneração do sistema nervoso central, resultando na perda progressiva das habilidades cognitivas ${ }^{22}$. Assim, as intervenções cognitivas não detêm o avanço da doença, mas apenas fazem com que o indivíduo mantenha seu nível de habilidades e de funcionamento por um maior período de tempo ${ }^{25}$.

Negovanska et al. ${ }^{19}$ investigaram a percepção do nível de ansiedade e observaram melhora significativa em comparação ao grupo controle. Estudos prévios ${ }^{26-28}$ mostraram que a redução do estresse percebido pelo paciente tem potencial para atrasar a institucionalização do paciente. Além disso, Santos et al. ${ }^{21}$ também evidenciaram redução no nível de agitação dos pacientes do grupo experimental após a intervenção, quando comparados ao grupo controle. Tais achados podem refletir não somente na saúde do paciente, mas também reduzir a carga de cuidados por parte dos familiares/cuidadores. 


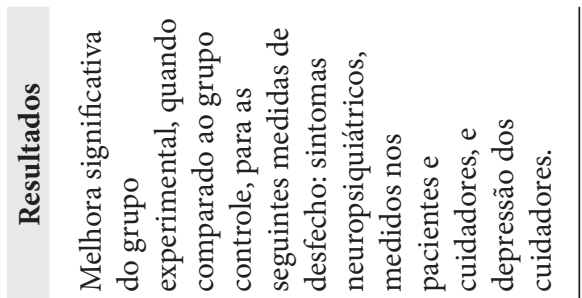
$\hat{\widehat{\Omega}} \stackrel{0}{3}$

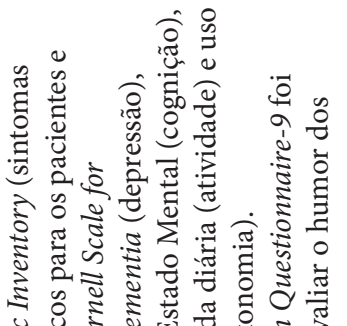
है के ₹ँ क्ष

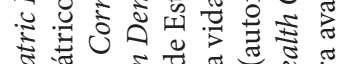

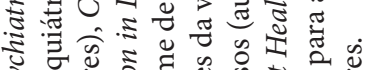

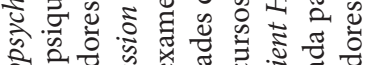
ڤิ

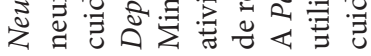

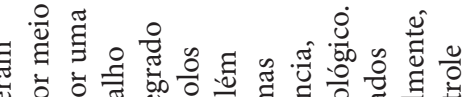

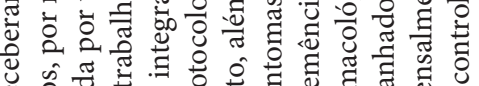

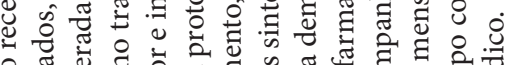

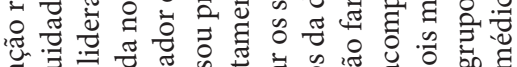

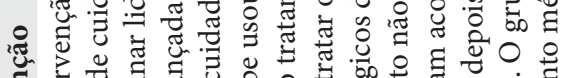

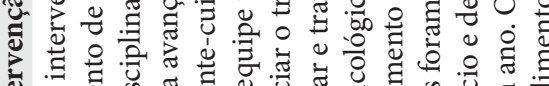

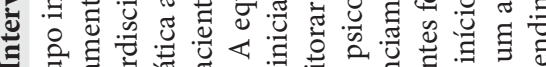

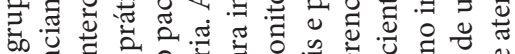

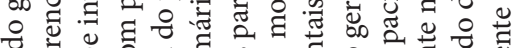

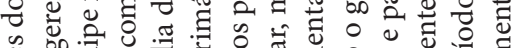

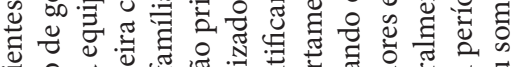

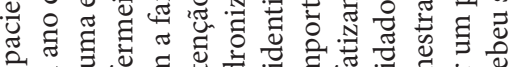

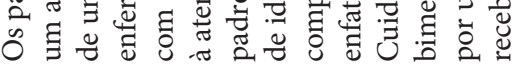
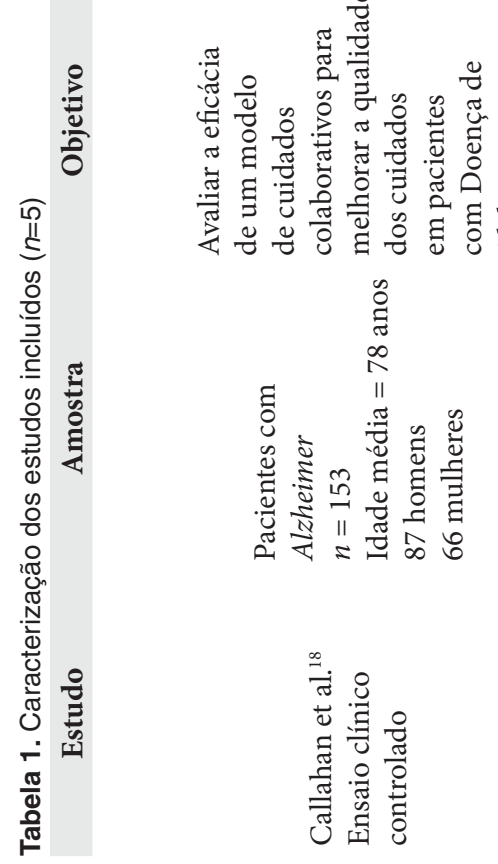
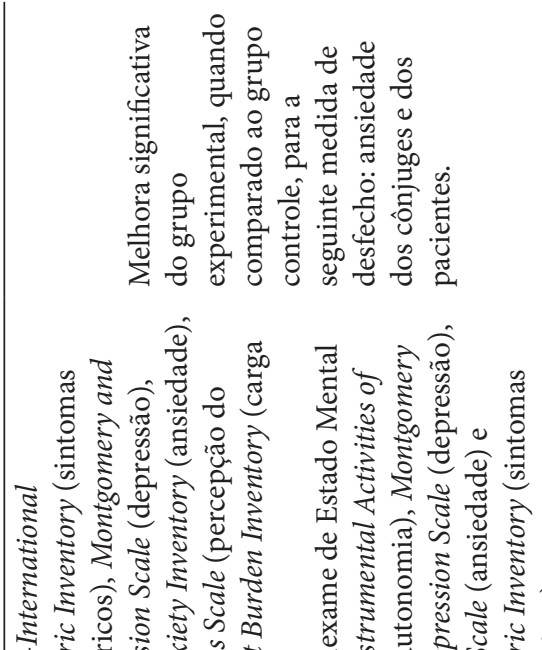

₹.

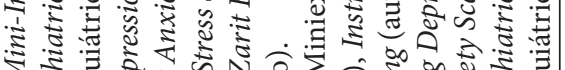

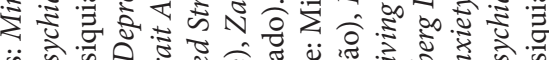

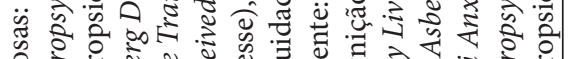

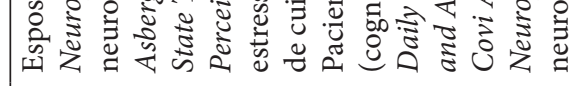
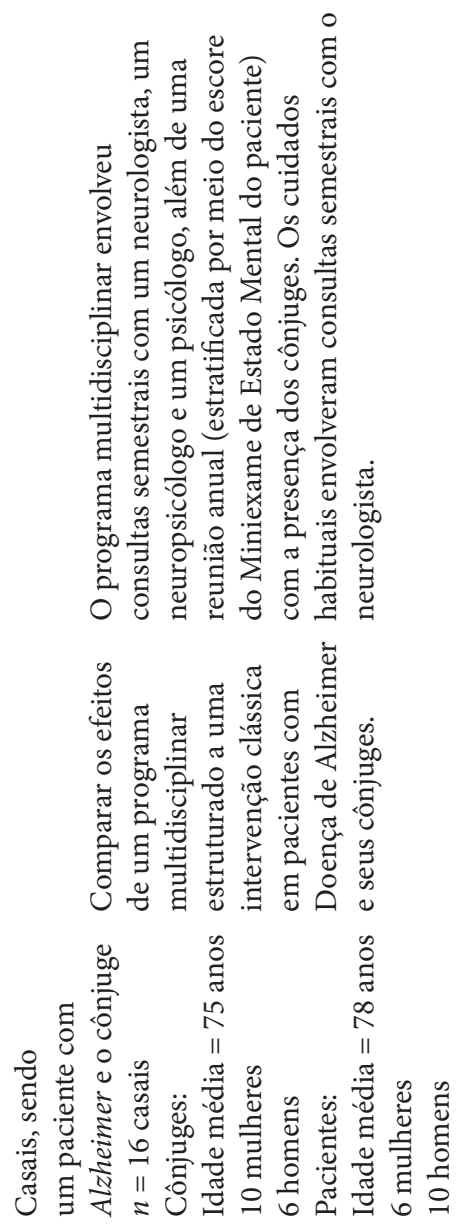
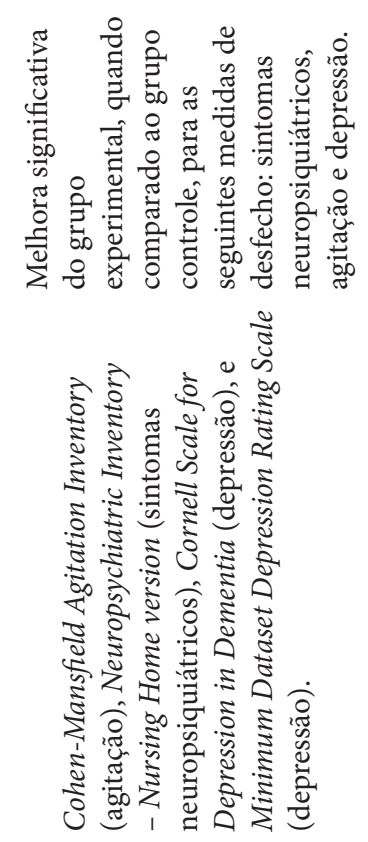

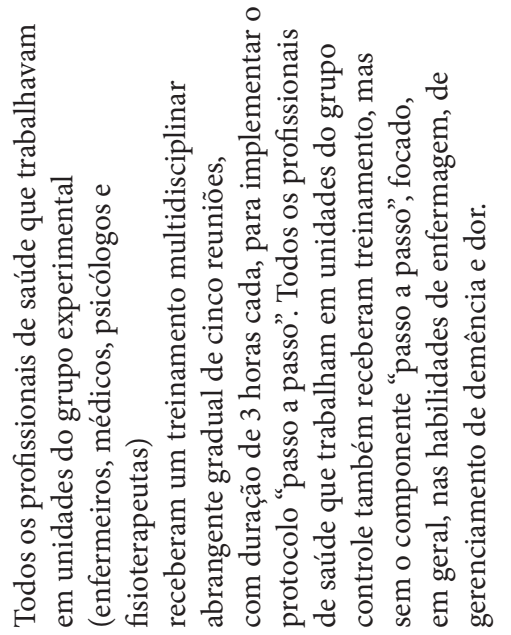

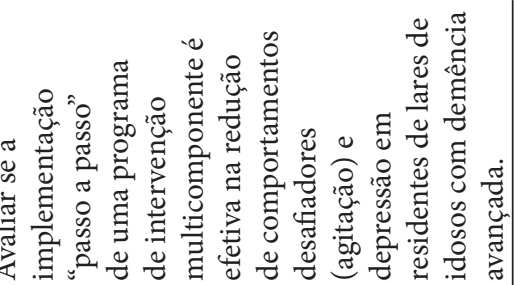
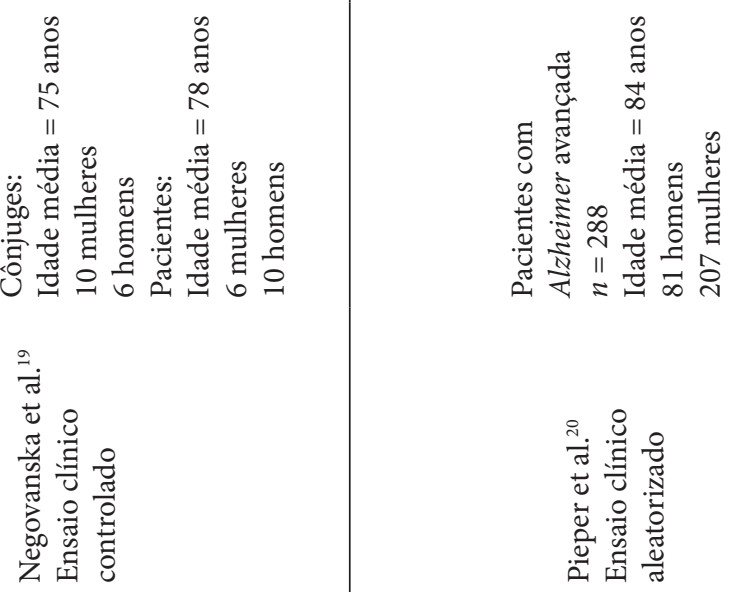

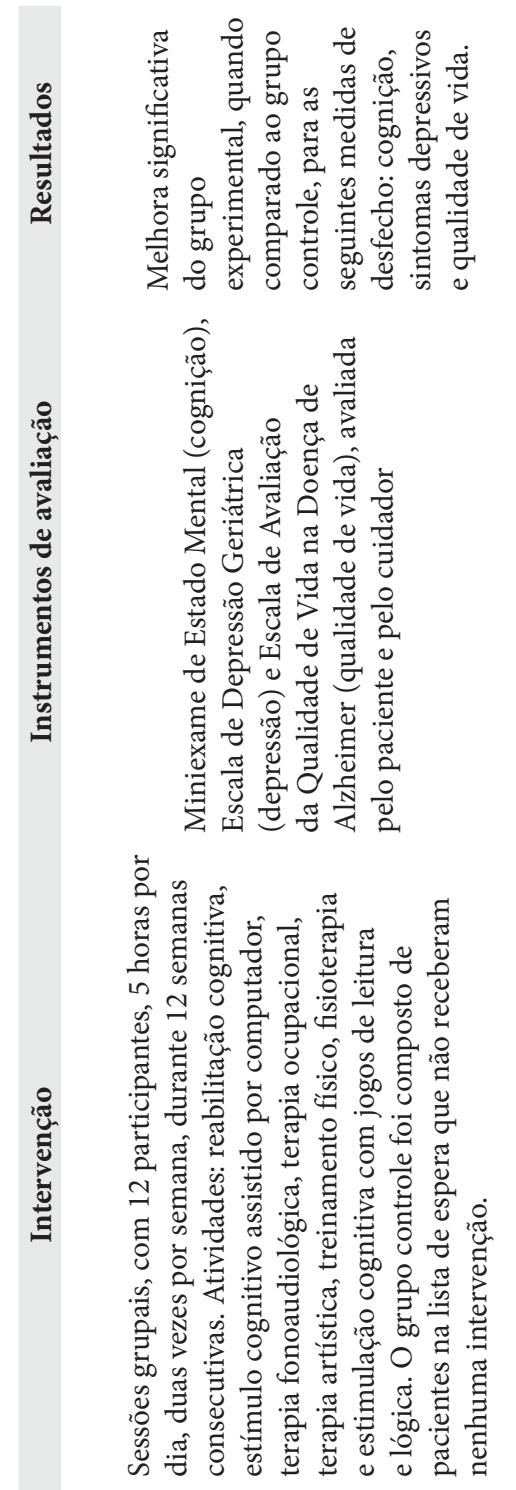

:

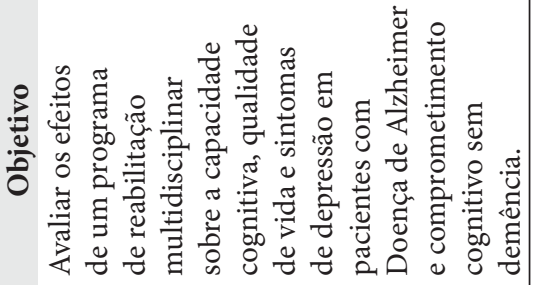

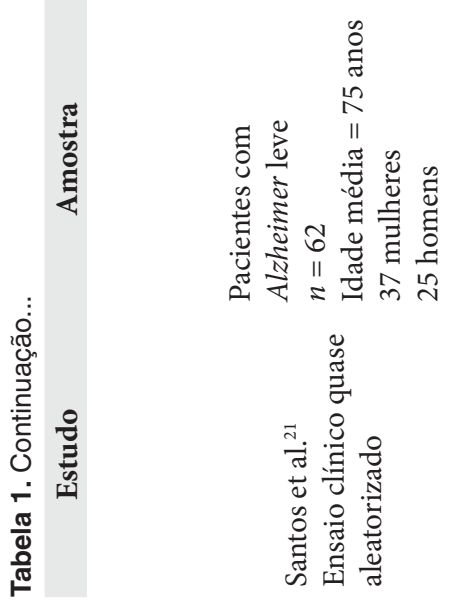

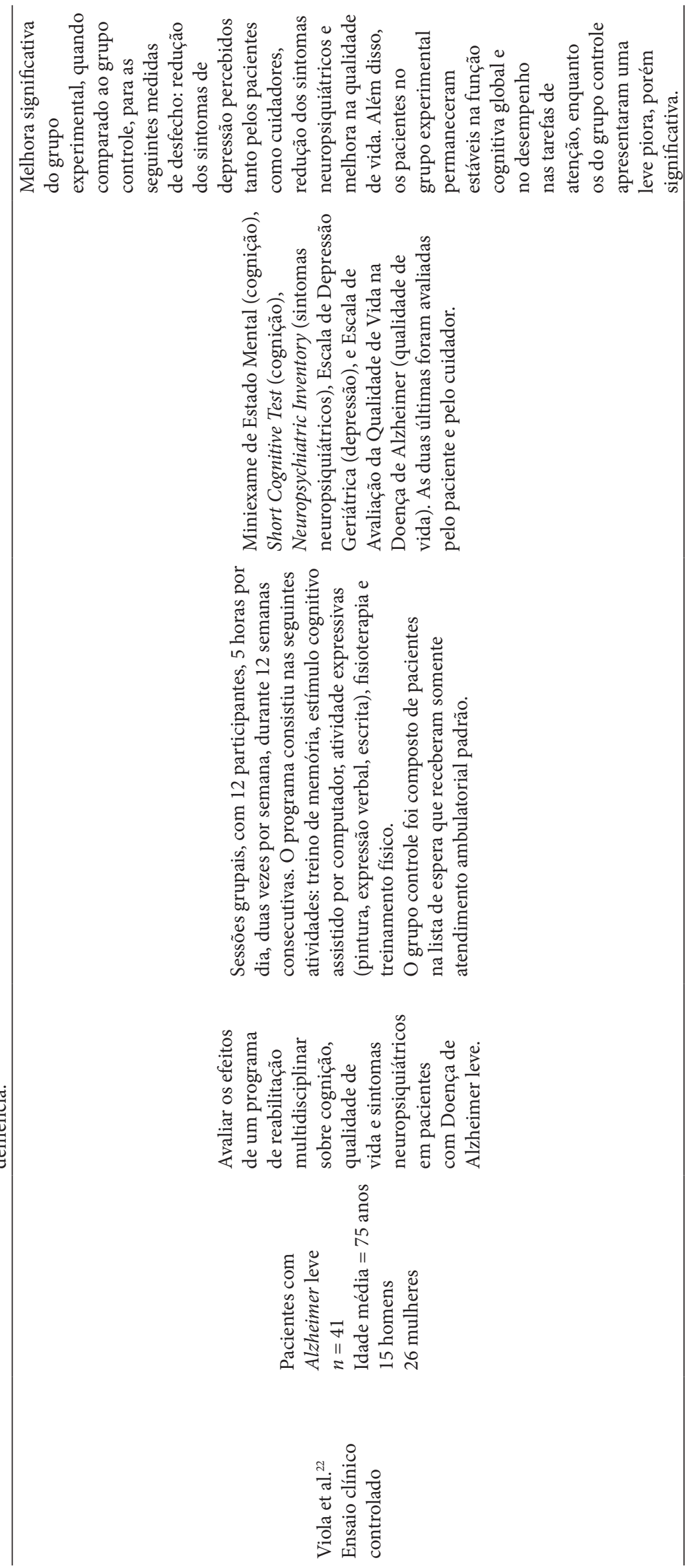


Tabela 2. Detalhamento dos estudos na escala PEDro

\begin{tabular}{|c|c|c|c|c|c|}
\hline CRITÉRIOS & Callahan et al. ${ }^{18}$ & Negovanska et al. ${ }^{19}$ & Pieper et al. ${ }^{20}$ & Santos et al. ${ }^{21}$ & Viola et al..$^{22}$ \\
\hline Aleatorização & $\mathrm{N}$ & $\mathrm{N}$ & S & S & $\mathrm{N}$ \\
\hline Cegamento da distribuição dos participantes & $\mathrm{N}$ & $\mathrm{N}$ & $\mathrm{N}$ & S & $\mathrm{N}$ \\
\hline Similaridade inicial entre os grupos & S & S & S & $\mathrm{N}$ & S \\
\hline Cegamento dos participantes & $\mathrm{N}$ & $\mathrm{N}$ & $\mathrm{N}$ & S & $\mathrm{N}$ \\
\hline Cegamento dos terapeutas & $\mathrm{N}$ & $\mathrm{N}$ & $\mathrm{N}$ & $\mathrm{N}$ & $\mathrm{N}$ \\
\hline Cegamento dos avaliadores & $\mathrm{N}$ & $\mathrm{N}$ & S & $\mathrm{N}$ & S \\
\hline Medidas de um desfecho primário & S & S & S & S & S \\
\hline Intenção de tratar & S & $\mathrm{N}$ & $S$ & $\mathrm{~N}$ & $\mathrm{~N}$ \\
\hline Comparação intergrupos do desfecho primário & S & S & S & S & S \\
\hline Medidas de precisão e de variabilidade & S & S & S & S & S \\
\hline Total & $5 / 10$ & $4 / 10$ & $7 / 10$ & $6 / 10$ & $5 / 10$ \\
\hline
\end{tabular}

$\mathrm{S}=\mathrm{Sim} ; \mathrm{N}=\mathrm{Não}$

Outros sintomas foram avaliados, relacionados à independência dessa população, à realização de atividades e à qualidade de vida $^{18,19,21,22}$. No entanto, não foram encontradas melhoras significativas no grupo experimental em relação ao grupo controle em nenhum dos estudos que investigaram as medidas de autonomia e a execução de atividades diárias ${ }^{18,19}$. No entanto, dois estudos que avaliaram os efeitos do tratamento multidisciplinar na qualidade de vida desses pacientes obtiveram melhora no grupo experimental comparado ao grupo controle ${ }^{21,22}$. É possível que a intervenção psicossocial se revele uma estratégia eficaz para melhorar a qualidade de vida de pacientes com DA. Durante o programa de reabilitação, os pacientes com DA podem aumentar sua socialização e criar uma rede de alianças e de ajuda mútua, o que pode reduzir a presença de sintomas depressivos e melhorar a percepção de qualidade de vida desses indivíduos ${ }^{22}$.

Por fim, Pieper et al..$^{20}$ investigaram a eficácia de um programa de intervenção multidisciplinar, quando implementado em uma metodologia "passo a passo" em pacientes com DA institucionalizados. Os autores reportaram resultados positivos para sintomas neuropsiquiátricos, agitação e depressão, em comparação com um grupo que não teve a implementação por essa metodologia ${ }^{20}$. Esses resultados demonstram a importância da implementação gradual, quando se trata da alteração da forma de cuidados dentro de uma instituição. O processo de mudança requer treinamento por parte do corpo profissional e familiarização do processo pelos pacientes.

Em relação aos cuidadores, melhoras significativas também foram observadas nos dois estudos que investigaram os efeitos do tratamento multidisciplinar na DA nessa população ${ }^{18,19}$. Os cuidadores são chaves principais no processo de cuidado e de evolução dos pacientes com demência, principalmente nas fases mais avançadas da doença ${ }^{18,19}$. Assim, melhorar aspectos, como ansiedade e depressão, desses indivíduos é fundamental para garantir um cuidado diário de qualidade para os pacientes com $\mathrm{DA}^{18,19}$.

Dos cincos artigos, apenas três deles descreveram a participação da fisioterapia no tratamento de pacientes com $\mathrm{DA}^{20-22}$. Embora ainda sejam limitados os ensaios clínicos sobre a fisioterapia e a DA, sabe-se que a prática de atividade física de pacientes com DA leva a uma melhora das habilidades motoras, da qualidade do sono e da circulação sanguínea e previne algumas lesões ortopédicas, além de o treino aeróbio também melhorar as funções mentais ${ }^{29,30}$. Assim, o tratamento fisioterapêutico passa a ter grande importância para retardar a progressão das perdas motoras, evitar encurtamentos e deformidades e incentivar a independência do indivíduo com Alzheimer ${ }^{29}$.

Uma das limitações desta revisão é a inclusão de apenas cinco estudos significativamente heterogêneos entre si, de qualidade metodológica moderada, principalmente o estudo de Negovanska et al. ${ }^{19}$, que alcançou nota 4 na escala PEDro. Além disso, vale ressaltar que alguns estudos com amostras pequenas e a utilização de protocolos de tratamento completamente diferentes entre si (de sessões semanais a acompanhamento anual, com diferentes modalidades profissionais) dificultam conclusões mais objetivas e definitivas. Por fim, outra limitação seria a não padronização das fases da doença, uma vez que dois dos estudos abordaram a DA leve, outros dois não especificaram a fase da doença e um teve como amostra pacientes com DA avançada. Dessa forma, mais estudos são necessários para investigar os benefícios do tratamento multidisciplinar em pacientes com DA. Ensaios clínicos randomizados, com amostras maiores, adequada qualidade metodológica, protocolos padronizados e detalhadamente descritos, investigando diferentes medidas de desfecho, comprovariam os possíveis benefícios desse tipo de abordagem para esses pacientes, fornecendo aos profissionais mais uma alternativa de tratamento eficaz.

Esta revisão sistemática evidenciou que o programa multidisciplinar pode ser eficaz no tratamento de pacientes com DA, com melhoras significativas, principalmente, em sintomas neuropsiquiátricos, depressão e qualidade de vida. No entanto, os resultados para cognição, nível de ansiedade, independência e realização de atividades não foram significativos ou foram conflitantes. 


\section{REFERÊNCIAS}

1. Veras RP. O anacronismo dos modelos assistenciais na área da saúde: mudar e inovar, desafios para o setor público e o privado. Rio de Janeiro: Instituto de Medicina Social, Universidade do Estado do Rio de Janeiro; 2000. (Série Estudos em Saúde Coletiva; no. 211).

2. Ávila R, Miotto EC. Funções executivas no envelhecimento normal e na doença de Alzheimer. J Bras Psiquiatr. 2003;52:53-63.

3. World Health Organization. Dementia: a public health priority. Geneva: WHO; 2012.

4. Bertazone TMA, Ducatti M, Camargo HPM, Batista JMF, Kusumota L, Marques S. Ações multidisciplinares/ interdisciplinares no cuidado ao idoso com Doença de Alzheimer. Rev Rene. 2016;17:144-53. http://dx.doi. org/10.15253/2175-6783.2016000100019.

5. Bottino CMC, Almeida OP. Demências: quadro clínico e critérios diagnósticos. In Almeida OP, Nitrini R, editores. Demências. São Paulo: Fundo Editorial Byk; 1995.

6. Da-Silva SL, Pereira DA, Veloso F, Satler CE, Arantes A, Guimarães RM. Programa de reabilitação neuropsicológica da memória aplicada à demência: um estudo não controlado intrasujeitos. Estud Psicol. 2011;28:229-40.

7. Engelhardt E, Brucki SMT, Cavalcanti JLS, Forlenza OV, Laks J, Vale FAC. Tratamento da Doença de Alzheimer: recomendações e sugestões do Departamento Científico de Neurologia Cognitiva e do Envelhecimento da Academia Brasileira de Neurologia. Arq Neuropsiquiatr. 2005;63(4):110412. http://dx.doi.org/10.1590/S0004-282X2005000600035. PMid:16400437.

8. Forlenza OV. Tratamento farmacológico da doença de Alzheimer. Rev Psiquiatr Clin (Santiago). 2005;32(3):137-48. http://dx.doi.org/10.1590/ S0101-60832005000300006.

9. Graff MJ, Adang EM, Vernooij-dassen MJ, Dekker J, Jönsson L, Thijssen $\mathrm{M}$, et al. Community occupational therapy for older patients with dementia and their care givers: cost effectiveness study. BMJ. 2008;336(7636):134-8. http://dx.doi.org/10.1136/bmj.39408.481898.BE. PMid:18171718.

10. Kim HJ, Yang Y, Oh JG, Oh S, Choi H, Kim KH, et al. Effectiveness of a community-based multidomain cognitive intervention program in patients with Alzheimer's disease. Geriatr Gerontol Int. 2015;16(2):191-9. http:// dx.doi.org/10.1111/ggi.12453. PMid:25656505.

11. Vale FAC, Corrêa YC No, Bertolucci PHF, Machado JCB, Silva DJ, Allam $\mathrm{N}$, et al. Treatment of Alzheimer's disease in Brazil: I. Cognitive disorders. Dement Neuropsychol. 2011;5(3):178-88. http://dx.doi.org/10.1590/S198057642011DN05030005. PMid:29213742.

12. Schneider $L$, Tariot $P$. Treatment of dementia. In Salzman C, editor. Clinical geriatric psychopharmacology. 3 ed. Baltimore: Williams \& Wilkins; 1998.

13. Luzardo AR, Gorini MIPC, Silva APSS. Características de idosos com doença de Alzheimer e seus cuidadores: uma série de casos em um serviço de neurogeriatria. Texto Contexto Enferm. 2006;15(4):587-94. http://dx.doi. org/10.1590/S0104-07072006000400006.

14. Araújo MBS, Rocha PM. Trabalho em equipe: um desafio para a consolidação da estratégia de saúde da família. Cien Saude Colet. 2007;12(2):455-64. http://dx.doi.org/10.1590/S1413-81232007000200022. PMid:17695079.

15. Herbert R, Jamtvedt G, Mead J, Hagen K. Practical evidence-based physiotherapy. New York: Butterwoth-Heinemann; 2011.

16. Padula R, Pires R, Alouche S, Chiavegato L, Lopes A, Costa L. Analysis of reporting of systematic reviews in physical therapy published in Portuguese. Braz J Phys Ther. 2012;16(4):281-9. http://dx.doi.org/10.1590/S141335552012005000040. PMid:22858736.

17. Physiotherapy Evidence Database [Internet]. Australia: PEDRro; 2017 [citado em 2017 jul 5]. Disponível em: www.pedro.org.au
18. Callahan CM, Boustani MA, Unverzagt FW, Austrom MG, Damush TM, Perkins AJ, et al. Effectiveness of collaborative care for older adults with Alzheimer disease in primary care: a randomized controlled trial. JAMA. 2006;295(18):2148-57. http://dx.doi.org/10.1001/jama.295.18.2148. PMid:16684985.

19. Negovanska V, Hergueta T, Guichart-Gomez E, Dubois B, Sarazin M, Bungener C. Beneficial effect of a cognitive behavioral and multidisciplinary program in Alzheimer Disease on spouse caregiver anxiety: French study ELMMA. Rev Neurol (Paris). 2011;167(2):114-22. http://dx.doi.org/10.1016/j. neurol.2010.07.002. PMid:20864140.

20. Pieper MJ, Francke AL, Van der Steen JT, Scherder EJ, Twisk JW, Kovach $\mathrm{CR}$, et al. Effects of a stepwise multidisciplinary intervention for challenging behavior in advanced dementia: A cluster randomized controlled trial. J Am Geriatr Soc. 2016;64(2):261-9. http://dx.doi.org/10.1111/jgs.13868. PMid:26804064.

21. Santos GD, Nunes PV, Stella F, Brum PC, Yassuda MS, Ueno LM, et al. Multidisciplinary rehabilitation program: effects of a multimodal intervention for patients with Alzheimer's disease and cognitive impairment without dementia. Arch Clin Psychiatry. 2015;42(6):153-6. http://dx.doi. org/10.1590/0101-60830000000066.

22. Viola LF, Nunes PV, Yassuda MF, Aprahamian I, Santos FS, Santos GD, et al. Effects of a multidisciplinar cognitive rehabilitation program for patients with mild Alzheimer's disease. Clinics. 2011;66(8):1395-400. http://dx.doi. org/10.1590/S1807-59322011000800015. PMid:21915490.

23. Hill JW, Futterman R, Duttagupta S, Mastey V, Lloyd JR, Fillit H. Alzheimer's disease and related dementias increase costs of comorbidities in managed Medicare. Neurology. 2002;58(1):62-70. http://dx.doi.org/10.1212/ WNL.58.1.62. PMid:11781407.

24. Mosher KI, Wyss-Coray T. Microglial dysfunction in brain aging and Alzheimer's Disease. Biochem Pharmacol. 2014;88(4):594-604. http:// dx.doi.org/10.1016/j.bcp.2014.01.008. PMid:24445162.

25. Correa SES, Silva DB. Abordagem cognitiva na intervenção terapêutica ocupacional com indivíduos com Doença de Alzheimer. Rev Bras Geriatr Gerontol. 2009;12(3):463-74. http://dx.doi.org/10.1590/1809-9823.2009.00012.

26. Nobili A, Riva E, Tettamanti M, Lucca U, Liscio M, Petrucci B, et al. The effect of a structured intervention on caregivers of patients with dementia and problem behaviors: a randomized controlled pilot study. Alzheimer Dis Assoc Disord. 2004;18(2):75-82. http://dx.doi.org/10.1097/01. wad.0000126618.98867.fc. PMid:15249851.

27. Spijker A, Vernooij-Dassen M, Vasse E, Adang E, Wollersheim H, Grol $\mathrm{R}$, et al. Effectiveness of nonpharmacological interventions in delaying the institutionalization of patients with dementia: a meta-analysis. J Am Geriatr Soc. 2008;56(6):1116-28. http://dx.doi.org/10.1111/j.1532-5415.2008.01705.x. PMid:18410323.

28. de Vugt ME, Riedijk SR, Aalten P, Tibben A, van Swieten JC, Verhey FRJ. Impact of behavioural problems on spousal caregivers: a comparison between Alzheimer's disease and frontotemporal dementia. Dement Geriatr Cogn Disord. 2006;22(1):35-41. http://dx.doi.org/10.1159/000093102. PMid:16679763.

29. Melo MA, Driusso P. Proposta Fisioterapêutica para os cuidados de Portadores da Doença de Alzheimer. Envelhec Saúd. 2006;12(4):11-18.

30. Gomes MVSS. Intervenção da educação física nos indivíduos com Doença de Alzheimer. Fisioter Bras. 2002;3(1):53-60.

Recebido em: Dez. 12, 2017

Aprovado em: Maio 30, 2018 


\section{APÊNDICE A}

MEDLINE

1. exp Dementia/

2. Delirium, Dementia, Amnestic, Cognitive Disorders/

3. dement*.mp.

4. alzheimer*.mp.

5. ("organic brain disease" or "organic brainsyndrome").mp.

6. "benign senescent forgetfulness".mp.

7. (cerebr* adj2 deteriorat*).mp.

8. (cerebral* adj2 insufficient*).mp.

9. or $/ 1-8$

10. rehabilitation.fs.

11. exp Rehabilitation/

12. exp Ambulatory Care/

13. exp Physical Therapy Modalities/

14. exp Home Care Services/

15. Inpatients/

16. Outpatients/

17. exp Behavior Therapy/

18. exp Social Work/

19. Dietetics/

20. exp Dietary Services/

21. exp Counseling/

22. exp Patient Care Team/

23. (multidisciplinary or multi-disciplinary or integrated or interdisciplinary or inter-disciplinary).mp.

24. (rehabilitat* or physiotherap* or physical therap* or speech or occupation* or social work*).mp.

25. (cognitive therap* or behavio?rtherap* or counsel?ing or nutrition* or diet* or food).mp.

26. (outpatient* or inpatient* or hospital* or home).mp.

27. multidisciplinar\$.mp.

28. interdisciplinar\$.mp.

29. multiprofessional\$.mp.

30. multimodal\$.mp

31. or/10-30

32. 9 and 31

\section{CINAHL}

S1 (MH "Dementiat")

S2 (MH "Delirium") or (MH "Delirium, Dementia, Amnestic, Cognitive Disorders")

S3 (MH "Wernicke's Encephalopathy")

S4 TX dement*

S5 TX alzheimer*

S6 TX lewy* N 2 bod* 
S7 TX deliri*

S8 TX chronic N2 cerebrovascular

S9 TX "organic brain disease" or "organicbrain syndrome"

S10 TX "normal pressure hydrocephalus"and "shunt*"

S11 TX "benign senescent forgetfulness"

S12 TX cerebr* N2 deteriorat*

S13 TX cerebral* N2 insufficient*

S14 TX pick* N2 disease

S15 TX creutzfeldt or jcd or cjd

S16 TX huntington*

S17 TX binswanger*

S18 TX korsako*

$\mathrm{S} 19 \mathrm{~S} 1$ or $\mathrm{S} 2$ or S 3 or S4 or S5 or S6 or S7or S8 or S9 or S10 or S11 or S 12 or S13 orS14 or S15 or S16 or S17 or S18

S20 (MH "Behavior Therapy+")

S21 (MH "Treatment Outcomest")

S22 "rehabilitation clinic*"

S23 (MH "Rehabilitation Centers+")

S24 (MH "Rehabilitation+")

S25 (MH "Rehabilitation, Vocationalt")

S26 (MH "Occupational Therapy+")

S27 (MH "Social Work+")

S28 "pain relief unit*" 5

S29 "pain service*" 161

S30 "pain centre*" 21

S31 "pain center*" 141

S32 (MH "Pain Clinics") 368

S33 (MH "Social Environment+") 24,968

S34 (MH "Support, Psychosocialt") 36,808

S35 (MH “Patient Education") 37,325

S36 "patient care management" 81

S37 (MH “Patient Centered Care") 11,891

S38 "patient care team" 63

S39 (MH "Combined Modality Therapy+") 16,090

S40 "multimodal" 1,509

S41multiprofessional 561

S42 (MH "Collaboration")

S43 "interdisciplinary"

S44 "multidisciplinary"

S45 (MH "Multidisciplinary Care Te am+")

S46 (MH "Support, Psychosocialt")

S47 "patient care management" 


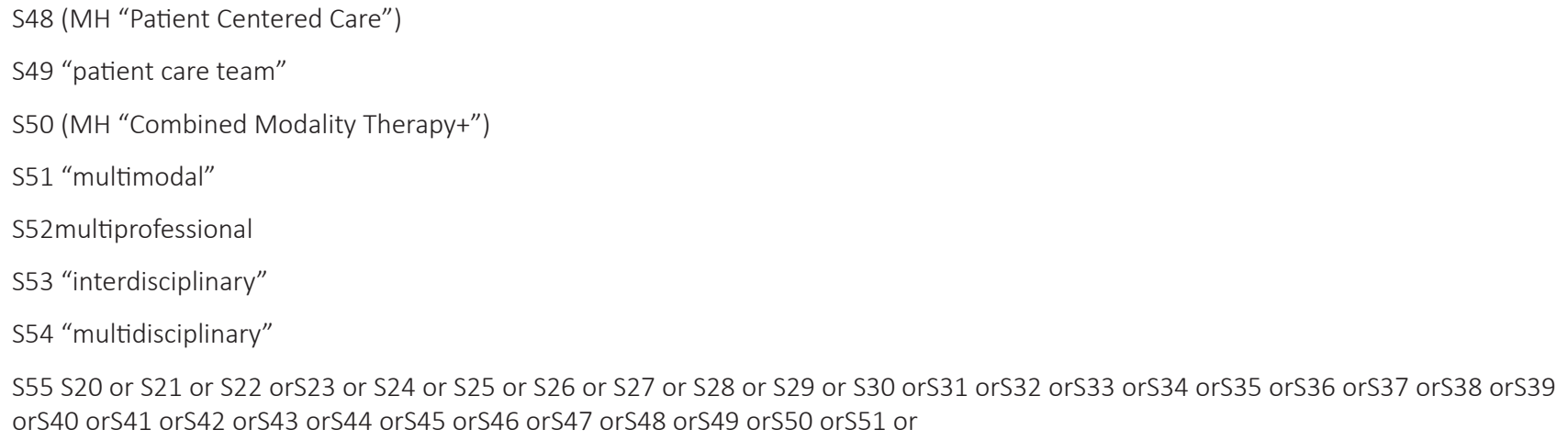

\section{WEB OF SCIENCE}

Topic=(dement* OR alzheimer* OR "lewybod*" OR DLB OR "vascular cognitive impairment*" OR FTD OR FTLD OR "cerebrovascular insufficienc $\left.{ }^{* \prime}\right)$ AND

Topic=(dement* OR alzheimer* OR "lewy bod*" OR DLB OR "vascular cognitive impairment*" OR FTD OF FTLD OR "cerebrovascular insufficienc*") AND

Topic=(rehabilitation OR "Social Environment" OR "patient care management" OR combined OR modalities OR therapies OR "Combined Modality Therapy" OR multimodal OR multiprofessional OR interdisciplinary OR multidisciplinary OR "Multidisciplinary Care Team")

LILACS

1. Alzheimer OR demencia OR dement OR lewy bod

2. (Mh Ambulator y Care) OR (Mh Rehabilitation) OR (Mh Hospitalization) OR (Mh Physical The r apy Modalities) O R (MhHome Care Services, Hospital-Based) OR (Mh Home Care Services) OR (Mh Inpatients) OR (Mh Outpatients) OR (MhCognitiveTherapy) OR (Mh Behavior Therapy) OR (MhSocialWork) OR (Mh Dietetics) OR (Mh Dietary Services) OR (Mh Counseling) OR(Mh Patient Care Team) OR (multidisciplinary) OR (intergrated) OR rehabilitat\$) O R (home health care) O R (physiotherap\$) OR(physical therap\$) OR (speech) OR (occupation\$) OR (social work) OR (cognitive therap\$) OR (behavior therap\$) OR (counse ling)OR (nutrition) OR (diet\$) OR (food) OR (outpatient\$) OR (inpatient\$) OR (hospital\$)

3. 1 and 2

\section{PEDro}

Abstract and title: Alzheimer OR dement OR demencia

Method: clinical trial 